\section{4-2.5 DISCUSSION: COMPLEXITY, SIMPLICITY AND EPIDEMIOLOGY}

doi:10.1136/jech.2011.142976b.18

N Pearce.* London School of Hygiene and Tropical Medicine, London, UK

It is difficult nowadays to open a popular science magazine, or a leading science journal, without reading about complexity, the approach to science that is expected to "define the scientific agenda for the 21st century." But this has had little influence on the theory and practice of epidemiology. Complexity is the study of complex adaptive systems, and they key concepts are: self-organisation, adaptation, upheavals at the edge of chaos, the unpredictability of the effects of small changes in the initial conditions, and the existence of simplicity at some levels while chaos exists at others. There are very few examples of the use of complexity theory in epidemiology - the main ones to date involve communicable diseasebut there are many examples of epidemiological problems for which complexity theory is relevant. In particular, a focus on the population level, and the socio-cultural context, does not necessitate the use of complexity theory, but it does make its value and potential more relevant. However, complexity theory doesn't fit with standard approaches to epidemiology. If we are not to be "prisoners of the proximate" then it will be necessary to develop new epidemiologic methods that are more appropriate for addressing the complexity of population health. These new methods will look less like a randomised controlled trial, and more like complex observational research such as evolutionary biology and cosmology. If we are going to address the major public health problems of the 21st century, then complexity theory is likely to play an important role.

\subsection{NEGLECTED CONDITIONS IN VULNERABLE GROUPS}

\section{Chair: Prof. Alan Fenwick, UK \\ 04-3.1 DISTANCE AND QUALITY OF CARE STRONGLY INFLUENCE CHOICE OF DELIVERY PLACE IN RURAL ZAMBIA: A STUDY LINKING NATIONAL DATA IN A GEOGRAPHIC INFORMATION SYSTEM}

doi:10.1136/jech.2011.142976b.19

'S Gabrysch, ${ }^{2} \mathrm{~S}$ Cousens, ${ }^{2} \mathrm{~J}$ Cox, ${ }^{2} 0$ Campbell. ${ }^{1}$ Ruprecht-Karls-Universität, Heidelberg, Germany; ${ }^{2}$ London School of Hygiene \& Tropical Medicine, London, UK

Introduction Maternal and perinatal mortality could be reduced if all women delivered in settings where skilled attendants can provide Emergency Obstetric Care (EmOC) if complications arise. Epidemiological research on determinants of skilled attendance at delivery has focused on household and individual factors, neglecting the influence of distance and quality of care, in part due to a lack of suitable data.

Methods Using a Geographic Information System (GIS), we linked national household data from the Zambian Demographic and Health Survey 2007 with national facility data from the Zambian Health Facility Census 2005 and calculated straight-line distances. Health facilities were classified by whether they provided Comprehensive EmOC, Basic EmOC, limited or substandard services. Multivariable multilevel logistic regression analyses were performed to investigate the influence of distance to care and quality of care on place of delivery for 3682 rural births.

Results Only a third of rural Zambian births occurred at a health facility, and half were to mothers living further than $25 \mathrm{~km}$ from a facility of Basic EmOC standard or better. As distance to the closest health facility doubled, the odds of facility delivery decreased by $29 \%$ ( $95 \%$ CI $14 \%$ to $40 \%$ ). Independently, each step increase in quality of care led to $26 \%$ higher odds of facility delivery (95\% CI $7 \%$ to $48 \%$ ).

Conclusion Lack of geographic access to quality EmOC is a key factor explaining why most rural deliveries in Zambia still occur at home without skilled care; this needs to be addressed to lower maternal and perinatal mortality. Linking datasets using Geographic Information System has great potential for future research.

\section{4-3.2 DETERMINANTS OF DOMESTIC VIOLENCE AGAINST WOMEN IN AN EASTERN SAUDI COMMUNITY}

doi:10.1136/jech.2011.142976b.20

Z Afifi. ${ }^{*}$ King Faisal University, College of Medicine, Al Ahsa, Eastern province, Saudi Arabia

Introduction The objective of this study is to identify the risk factors underlying the occurrence of DV in an eastern Saudi community.

Methods The study included 2000 ever-married women aged 15-60 years while attending 10 randomly selected primary healthcare centers in Al Ahsa-KSA. Data were gathered through interviews using structured Arabic questionnaire. It included enquiry about the lifetime occurrence of violence, its types and determinants (personal, sociodemographic, paternal, maternal and other risk factors). Bivariate analysis was carried. Four Multivariate logistic regression (MLR) models were fitted to identify the significant risk factors in each type of violence.

Results The prevalence of overall, mental, physical and sexual violence is $39.3 \%, 35.9 \%, 17.9 \%$ and $6.9 \%$ respectively. The significant determinants of overall violence (MLR) are urban residence, wife's parental problems; husband problems with police and wife, smoking, fighting with others, being a victim of violence as a child, observed his mother being abused, and not spending enough time with family. The presence of each form of violence was significantly associated with the occurrence of others $(p=0.000)$. MLR revealed differences in the determinants of each type of violence. Marital discord was significant in all forms. Alcoholism and having problems with police only associated with sexual abuse. Protective factors are education except in postgraduate women, higher income status, and spending more time with family.

Conclusion Educating couples and increasing public awareness about risk factors is recommended. Healthcare providers are to follow high risk approach in identifying women exposed to violence based on identified factors.

\section{4-3.3 ACCURACY OF CLINICAL AND LABORATORY SIGNS FOR DENGUE DIAGNOSIS}

doi:10.1136/jech.2011.142976b.21

R P Daumas, P Brasil, R de V Carvalhaes Oliveira, B B G Carvalho, C Bressan, I Georg, R Nogueira, M Wakimoto, D V Carneiro, S R Lambert Passos. * Oswaldo Cruz Foundation, Rio de Janeiro/Rio de Janeiro, Brazil

Background In many endemic regions, serological tests for dengue are unavailable and diagnosis relies solely on clinical signs and basic laboratory workup.

Objective To identify clinical signs and basic haematological laboratory results potentially useful to distinguish dengue from other febrile illnesses.

Methods Prospective study in an outpatient setting in Rio de Janeiro from 2005 to 2008. Subjects included patients $>12$ years of age referring fever with duration $\geq 7$ days (acute fever) and without evident focuses of infection. Logistic regression analysis was used to identify symptoms, physical signs and haematological features accurate for the dengue diagnosis in patients evaluated between days $0-3$ and days $4-7$ from fever outset. 